\title{
Stereoelectroencephalography-guided radiofrequency thermocoagulation in drug-resistant focal epilepsy
}

\author{
Le Wang ${ }^{1 \#}$, Weipeng Jin ${ }^{2 \#}$, Yan Zhang ${ }^{1}$, Shimin Wang ${ }^{3}$, Qingyun $\mathrm{Li}^{3}$, Jie Qin ${ }^{3}$, Zhitao Li ${ }^{2}$, Yifeng Cheng ${ }^{2}$, \\ Keke Feng ${ }^{2}$, Shaoya Yin ${ }^{2,4}$ \\ ${ }^{1}$ Clinical College of Neurology, Neurosurgery and Neurorehabilitation, Tianjin Medical University, Tianjin, China; ${ }^{2}$ Department of Functional \\ Neurosurgery, Huanhu Hospital, Tianjin, China; ${ }^{3}$ Department of Neurology, Huanhu Hospital, Tianjin, China; ${ }^{4}$ Functional Neurosurgery \\ Laboratory of Tianjin Neurosurgical Institute, Tianjin, China \\ Contributions: (I) Conception and design: L Wang, S Yin; (II) Administrative support: None; (III) Provision of study materials or patients: S Yin; (IV) \\ Collection and assembly of data: L Wang, Y Zhang, W Jin; (V) Data analysis and interpretation: L Wang, S Yin, W Jin, S Wang, Q Li, J Qin; (VI) \\ Manuscript writing: All authors; (VII) Final approval of manuscript: All authors. \\ \#The authors contributed equally to this work. \\ Correspondence to: Prof. Shaoya Yin, MD. Department of Functional Neurosurgery, Huanhu Hospital, Tianjin, No. 6, Jizhao Road, Tianjin, China; \\ Tianjin Neurosurgical Institute, Tianjin 300350, China. Email: yinsya@hotmail.com.
}

Background: Stereoelectroencephalography (SEEG) has become a common diagnostic method in epilepsy surgery and is found to be safe for a wide range of clinical applications. SEEG combined with radiofrequency thermocoagulation (RF-TC) not only reveals the seizure onset zone by hypothesis, but also acts as a treatment option without any additional cost to institutions and patients. Thus, we analyzed the treatment of the SEEG-guided RF-TC.

Methods: This retrospective study analyzed seventeen epileptic patients treated with RF-TC between April 2019 and December 2020. All patients underwent a single round of SEEG-guided RF-TC treatment after more than three habitual seizures were recorded. The demographic characteristics of the patients were retrospectively reviewed. Outcomes were assessed using the Engel classification system.

Results: All patients underwent SEEG-guided RF-TC without catastrophic functional damage. Followup data of all patients were complete. The number of contacts per patients where RF-TC was applied ranged from 9 to 43 (mean: $17.7 \pm 10.2$ ). After RF-TC, the types of anti-epileptic drugs used reduced from $2.4 \pm 0.7$ to 1.6 \pm 0.7 . With RF-TC alone, four (23.5\%) patients achieved Engel Ia, two (11.8\%) patients achieved Engel $\mathrm{Ib}$, one patient underwent resection without seizure at the 5-month follow-up, five patients had a relapse after 3-10 months of seizure freedom, and five patients had recurrence after 1 month. After RF-TC, six patients underwent secondary interventions followed by resection. Overall, 12 patients achieved Engel Ia or Ib, three patients achieved Engel IIa or IIb, and two patients achieved Engel IIIa. There were no Engel IV cases.

Conclusions: SEEG-guided RF-TC performed in our institution was found to be a safe ablation procedure for the treatment of drug-resistant focal epilepsy. All patients experienced a reduction in the frequency of seizures after receiving RF-TC. RF-TC can be used as a palliative treatment option for patients with epilepsy who refuse surgery or cannot undergo resection surgery. Recurrence of focal epilepsy after RFTC can be treated with resection surgery to achieve the seizure-free status.

Keywords: Stereoelectroencephalography (SEEG); radiofrequency thermocoagulation (RF-TC); drugresistant epilepsy; focal epilepsy

Submitted Nov 30, 2021. Accepted for publication Feb 18, 2022.

doi: 10.21037/atm-21-6851

View this article at: https://dx.doi.org/10.21037/atm-21-6851 


\section{Introduction}

Stereoelectroencephalography (SEEG) was originally invented as a diagnostic tool by Talairach and Bancaud of St. Ann's Hospital in Paris in the 1960s. Implantation of deep electrodes by stereotaxic techniques, almost all the different areas of the cerebral cortex can be explored. After decades of development, SEEG surpassed the subdural electrode which is one of the most commonly used surgical evaluation methods for drug-resistant epilepsy (failure of adequate trials of two tolerated, appropriately chosen and used antiepileptic drug schedules to achieve sustained seizure freedom) in clinics (1). Presently, SEEG is used in diagnosis as well as treatment in combination with radiofrequency thermocoagulation (RF-TC) (2).

Nowadays, the common treatments for epilepsy include antiepileptic drug therapy, epileptogenic foci resection, neuromodulation, and so on. Stereotactic lesioning is not a new concept in epilepsy surgery; however, SEEG-guided RF-TC was first described by Guenot et al. in 2004 (3). RFTC is often performed after sufficient habitual seizures are recorded during SEEG monitoring with no additional costs. The diffusion pathway of seizures can be analyzed using SEEG; however, to further clarify the hypothesis regarding the epileptogenic focus, RF-TC should be performed. Some patients with epilepsy and limited epileptogenic foci can achieve seizure freedom through RF-TC alone. At present, the number of studies on RF-TC guided by SEEG is very small, and its efficacy needs to be further verified. This study explored the treatment outcomes of SEEG-guided RF-TC in drug-resistant focal epilepsy by retrospectively analyzing the clinical data of the patients with epilepsy. We present the following article in accordance with the STROBE reporting checklist (available at https://atm. amegroups.com/article/view/10.21037/atm-21-6851/rc).

\section{Methods}

\section{Patient data}

Seventeen patients (11 men and 6 women) with a confirmed diagnosis of drug-resistant focal epilepsy who underwent SEEG-guided RF-TC at the Functional Neurosurgery Department of Huanhu Hospital (Tianjin, China) between April 2019 and December 2020 were enrolled in this retrospective study. The following demographic data of the patients were extracted and analyzed: age, sex, age at seizure onset, age at diagnosis, past history, procedures prior to RF-TC, number of antiepileptic drugs (AEDs) used before and after RF-TC, seizure onset zone (SOZ) by hypothesis, number of contacts used in RF-TC, RF-TC outcomes (such as seizure recurrence after RF-TC), repeat operation after RF-TC, and Engel Class. All procedures performed in this study involving human participants were in accordance with the Declaration of Helsinki (as revised in 2013). The study was approved by ethics board of Tianjin Huanhu Hospital (No. 2021-059). Individual consent for this retrospective analysis was waived.

\section{Electrode implantation design}

All patients underwent a comprehensive evaluation that included seizure data analysis, semiology, long-term scalp video electroencephalography structural imaging studies [computed tomography (CT), 3-dimensional (3D) T1-weighted magnetic resonance imaging (MRI), T2-weighted MRI, fluid-attenuated inversion recovery MRI, susceptibility-weighted imaging, and 18 fluoro2-deoxyglucose positron emission tomography], and neuropsychological testing. Based on the "anatomoelectro-clinical correlations" of seizures, a multidisciplinary electroclinical hypothesis was formulated. Unlike the electrode implantation in RF-TC, the electrode scheme is guided by clarifying the epileptogenic area and resection boundary to ensure that the distance between electrodes is large $(10-20 \mathrm{~mm})$. The preoperative planning was completed using the Sinoplan system (SINOVATION, China). All patients underwent magnetic resonance angiography (MRA) and magnetic resonance venography (MRV), data of which were combined and used for electrode planning.

\section{Electrode implantation and seizure investigation}

Prior to electrode placement, hairs were shaved. CT scan was performed using the Leksell stereotactic headframe (ELKTA, Sweden) to obtain position information. Electrode implantation is performed under local anesthesia unless the patient does not cooperate. We used electrodes from SINO ROBOTICS (Beijing, China). Standard electrodes have 6 or 16 contacts. Each contact is $2 \mathrm{~mm}$ in length and $0.8 \mathrm{~mm}$ in diameter. The intercontact space is $1.5 \mathrm{~mm}$. Postoperatively, CT images were obtained to determine the accuracy of anatomic placement of electrode and the exact location of each contact on the electrode. Seizures were recorded in the standard fashion using the epilepsy monitoring unit (EMU). To shorten the duration 
of monitor, patients could reduce or withdraw AEDs to induce spontaneous seizures.

\section{RF-TC and removal of SEEG electrodes}

RF-TC was performed after more than three habitual seizures were recorded. The SOZ and involved contacts were determined through multidisciplinary discussion. The technique itself is quite simple. However, it was manipulated in the EMU and patients kept awake in the process. The parameters of the RF device (Model No. R2000B-M1, BNS, Beijing, China) were set as follows: $7.5 \mathrm{~W}$ power applied in two sessions of 30 seconds each, with a 30-second pause between the sessions. The total treatment time was 90 seconds. The current was passed across the two selected adjacent contacts and the changes in patient's language, movement, and other functions were observed throughout the process. This process was repeated for all planned contacts. Occasionally, the patient reported some discomfort, such as electromagnetic sound, heat sensation, and mild pain, which dissipated immediately after the passage of current ceased. One patient experienced seizures during RF-TC and the operation was stopped immediately. The patients underwent additional recording after TC to evaluate the effects of RFTC prior to electrode removal. After RF-TC was completed, the electrodes used in the EMU were removed without anesthesia. After removing the electrodes, the wound was blocked with a mupirocin ointment. Sutures were not required. Thereafter, head MRI was assessed within 48 hours. The imaging data and long-term SEEG monitoring images of typical case before and after RF-TC are shown in Figure 1.

\section{Follow-up}

Outcomes such as seizure recurrence after RF-TC, repeat operation after RF-TC, and Engel Class were assessed through telephone calls and outpatient visits.

\section{Statistical analysis}

Statistical analysis was performed using counts and percentages.

\section{Results}

\section{Patient demographics}

The age of 17 patients ( 11 men and 6 women) ranged from 13 to 46 years (mean age: $28.5 \pm 9.6$ years). The onset age of seizures ranged from 4 to 42 years (mean age, $19.7 \pm 11.2$ ). One patient (No. 6) had a previous history of CO intoxication, and one patient (No. 13) had a previous history of head trauma or meningitis. Two patients had a history of craniotomy, of which one had cerebral hemorrhage (No. 14) and the other had meningioma (No. 15). Before RF-TC, one patient underwent epileptogenic zone (EZ) resection (No. 2) and one patient underwent gamma knife treatment (No. 11). The seizure frequency varied from daily to monthly. All patients used more than three AEDs during the course of epilepsy. The average types of AEDs used before SEEG were $2.4 \pm 0.7$ that decreased to $1.6 \pm 0.7$ after RF-TC. The hypothetical SOZs were distributed in the frontal lobe (4 cases), cingulate gyrus ( 2 cases), and temporal lobe (11 cases). The main demographic characteristics of the patients are presented in Table 1.

\section{Seizure outcome}

All 17 patients underwent SEEG-guided RF-TC without catastrophic functional damage. Follow-up data of all cases were complete. The number of contacts used in RF-TC per patient ranged from 9 to 43 (mean: $17.7 \pm 10.2$ ). After RF-TC, the types of AEDs used reduced from $2.4 \pm 0.7$ to $1.6 \pm 0.7$, which was accompanied by the disappearance or remission of seizure. With RF-TC alone, four (23.5\%) patients achieved Engel Ia, two (11.8\%) patients achieved Engel $\mathrm{Ib}$, one patient underwent resection without seizure at the 5-month follow-up, five patients had a relapse after 3-10 months of seizure freedom, and five patients had recurrence after 1 month. Six patients underwent secondary interventions after RF-TC followed by resection. Overall, 12 patients achieved Engel Ia or Ib, three patients achieved Engel IIa or IIb, two patients achieved Engel IIIa. There were no Engel IV cases.

\section{Discussion}

Epilepsy is the most common serious chronic neurological disease, affecting more than 50 million people, with $80 \%$ of the patients with epilepsy living in low-income countries (4). The lives of patients with epilepsy are disrupted by seizures, comorbidities, and side effects of AEDs (5). The development of epilepsy surgery has resulted in reduction of seizures and improvement of the quality of life of many patients with drugresistant epilepsy. The EZ in most patients with focal epilepsy can be identified through non-invasive assessments (6). However, in some patients with epilepsy, the location of 

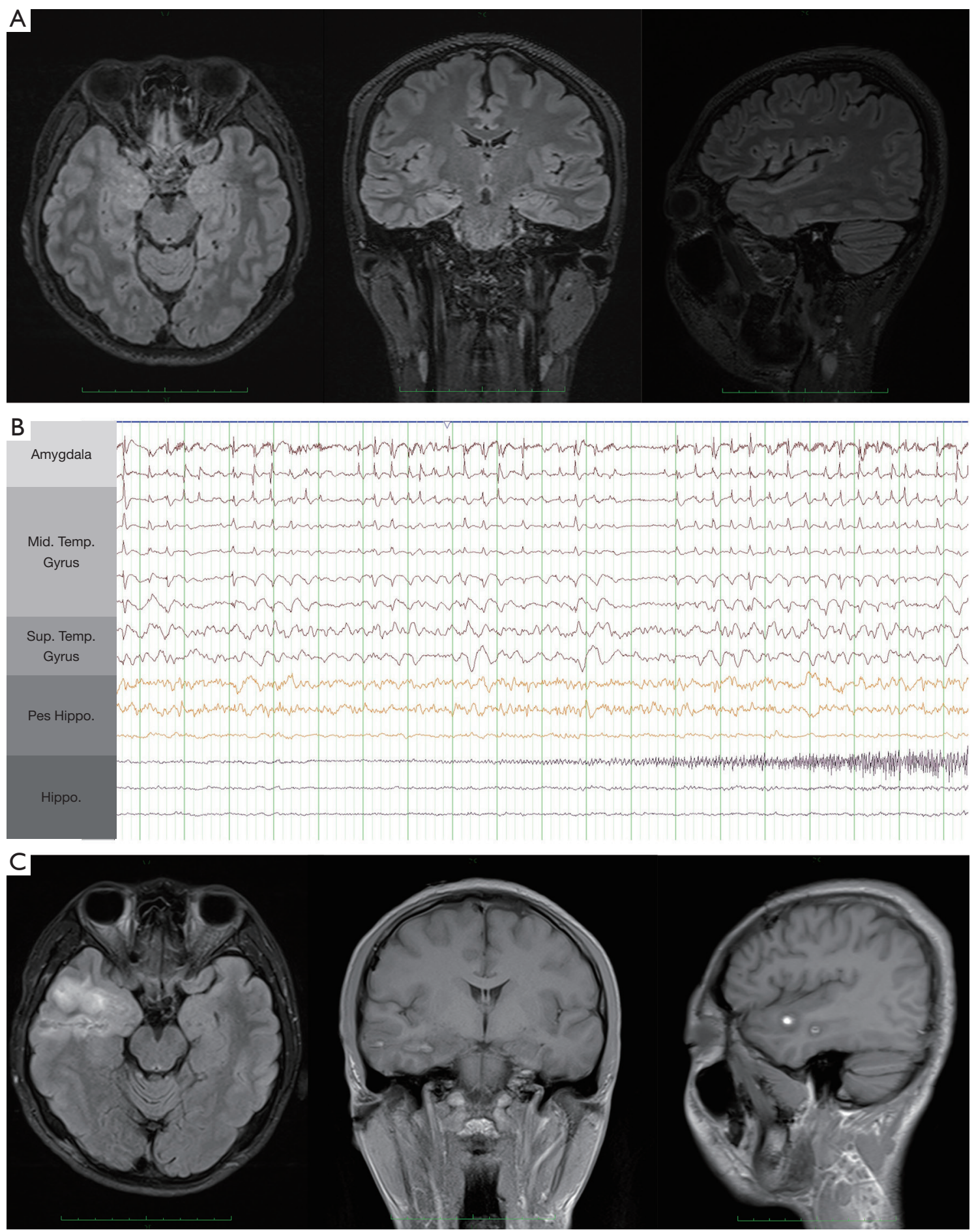

Figure 1 Example of RF-TC in a drug-refractory epilepsy case. (A) Representative case of drug-refractory epilepsy treated with RF-TC (No. 3). MR image before stereoelectroencephalography; (B) the onset of seizure captured by stereoelectroencephalography (Sup. Temp. Gyrus: superior temporal gyrus; Mid. Temp. Gyrus: middle temporal gyrus; Hippo.: hippocampus); (C) MR image obtained within 48 hours after RF-TC. RF-TC, radiofrequency thermocoagulation. 


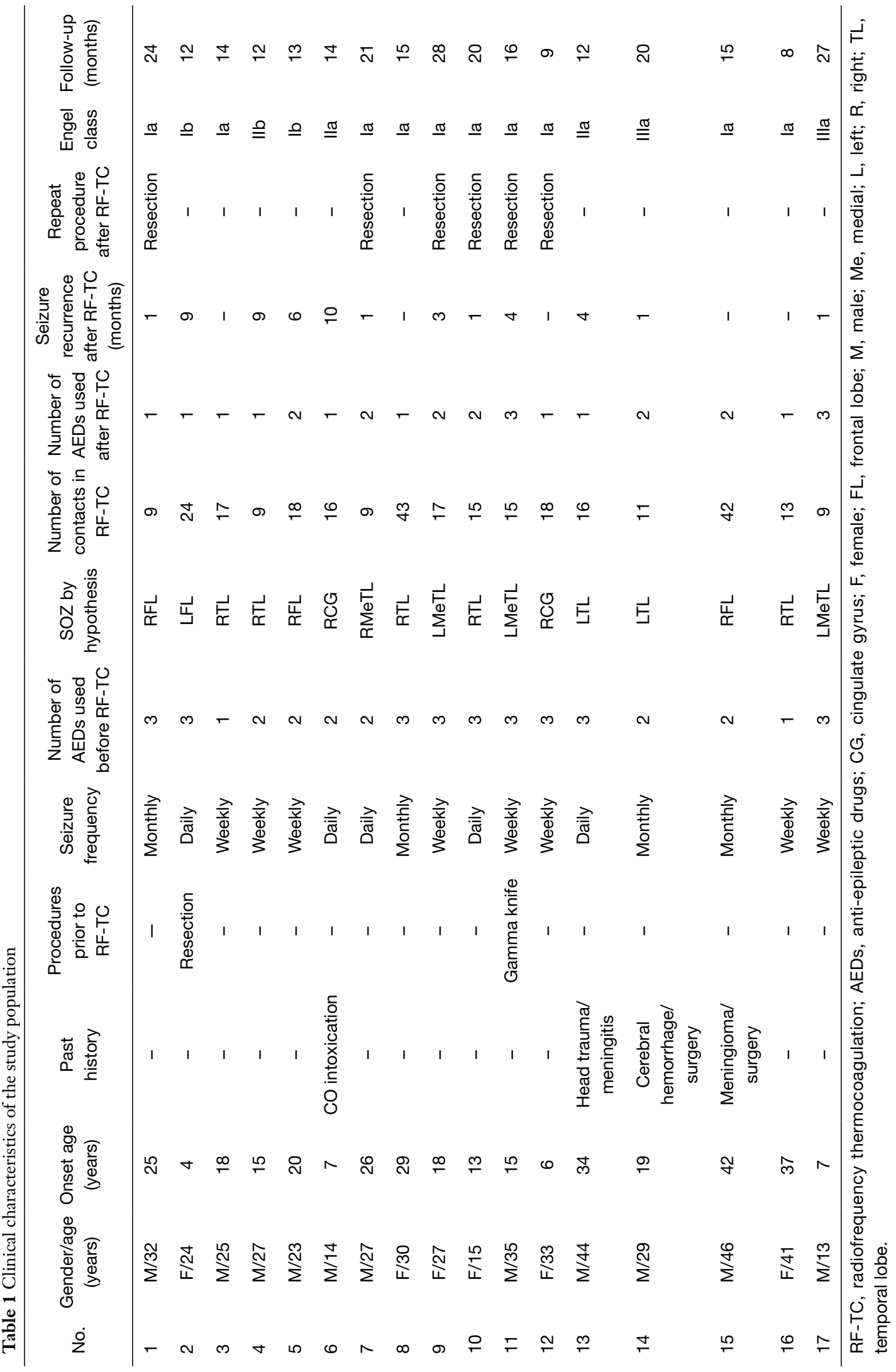


the $\mathrm{EZ}$ is contradictory or the $\mathrm{EZ}$ is closely related to the functional zone; therefore, the $\mathrm{EZ}$ cannot be safely removed, requiring further invasive evaluation.

The SOZ can be identified using SEEG by inserting electrodes deeper to simultaneously monitor multiple brain regions, especially the deep brain structures such as the hippocampus, amygdala, and hypothalamic hamartoma. Advantages, therefore, their application is gradually widespread (1). SEEG-guided RF-TC was first publicly reported in 2004 (3). The advantage of SEEG-guided RFTC lies in the use of SEEG monitoring electrodes for thermocoagulation, which avoids the risk of secondary implantation of electrodes. This study retrospectively analyzed the data of 17 patients with focal drug-refractory epilepsy who underwent SEEG-guided RF-TC and discussed the safety and efficacy of RF-TC in patients with epilepsy.

RF-TC denatures proteins by heat transfer to achieve local tissue damage. Current research has found that the damage in thermocoagulation is related to two factors: radiofrequency parameters and dipole selection. The thermosetting parameters recommended by the French guidelines differ from the original parameters (7). Initially, a study used fixed parameters for thermocoagulation (3); however, a recent study that used of unfixed parameters for thermocoagulation reported better results (8). Therefore, non-fixed parameters with power $(\mathrm{W})$ as the set value are currently used for thermocoagulation. In our institution, the thermocoagulation parameters are as follows: $7.5 \mathrm{~W}$ is applied in two thermocoagulation sessions, with each thermocoagulation session lasting 30 seconds. Thermocoagulation is performed without anesthesia. During the thermocoagulation process, the patient's state and occurrence of neurological damage are closely observed to prevent functional damage by immediately terminating the operation in adverse scenario. The French SEEG guidelines recommend the construction of dipoles for thermocoagulation (2). Different epilepsy centers adopt different methods to select contacts for constructing dipoles, although construction of dipoles by selecting adjacent electrodes for thermocoagulation was proven safe previously $(9,10)$. However, considering the large power used in thermocoagulation, we used adjacent contacts to construct a dipole for thermocoagulation. In this study, 17 patients who underwent RF-TC did not have permanent neurological impairment, indicating that performing RF$\mathrm{TC}$ with our parameters was highly safe in practice.

The effect of stereotactic thermocoagulation is controversial, and its efficacy in the literature varies greatly (6-63\%) (11). SEEG-guided RF-TC has a significant effect on epilepsy caused by hypothalamic hamartoma and FCD II $(9,12)$. This is not surprising because physicians are often attracted by MRI visible lesions and focus on them when treating epilepsy. The hypothalamic hamartoma is not required to be monitored by SEEG, and better results can be obtained only by lesion-guided stereotactic RF-TC (L-RFTC) (13), Although the use of L-RFTC may not be able to identify epileptic lesions, especially also in the presence of seizure forms other than laughter seizures (9). The thermocoagulation range is determined based on the SEEG results before RF-TC for FCD II; however, unlike other lesions, the record of the onset of seizures is not required to determine the thermocoagulation range in FCD II, only intermittent discharges are sufficient (14). Overall, the efficacy of SEEG-guided RF-TC alone is limited, especially in patients with MRI feminine (15). Mirza et al. analyzed 14 patients with drug-refractory epilepsy and reported that four patients had seizure remission maintained for $>12$ months after thermocoagulation and 11 patients had a relapse within 18 months (16). Dimova et al. (12) retrospectively analyzed the data of 23 patients with focal epilepsy who received RF-TC and found that at 32 months (2-119 months) of follow-up, 8 patients had a $>50 \%$ reduction in seizures after thermocoagulation, one patient had complete remission, and 15 (65.2\%) patients did not benefit from thermocoagulation. Bourdillon et al. (15) conducted a meta-analysis of 296 patients who underwent SEEG-guided RF-TC. The seizure-free rate after thermocoagulation was $23 \%$, and the seizure frequency was reduced by $>50 \%$ in $58 \%$ of the patients (15). The SEEG electrode design in this study aimed to locate the epileptic area and determine the scope of resection. Although the electrode implantation density was increased in the lesion area, there were no cases of pursuing complete destruction. However, 7 patients were seizure free for $>6$ months after RF-TC, of which 3 had seizure remission for $>1$ year. Two patients received RF-TC treatment for recurring seizures after epileptic foci resection (No. 2) and gamma knife treatment (No. 11), resulting in seizure remission for 9 and 4 months, respectively. All patients with epilepsy who underwent resection surgery (6/17 patients) achieved seizure freedom. The main reason for resection surgery was recurrence after RF-TC (5/6 patients).

Unlike the removal of epileptic foci in resection surgery, SEEG-guided RF-TC works by disrupting the epileptic network. SEEG electrodes should be implanted in the SOZ 
and the key nodes of the diffusion network. Cutting off the epileptic network by destroying limited contacts in RF-TC is considered ideal. However, current technical limitations make it is impossible to accurately identify all the key nodes of the onset and spread of the network. Based on the scope of the damaged epileptic network, SEEG-guided RFTC can achieve different effects such as complete absence of seizures, reduction of seizures, and transformation of drug-resistant epilepsy into drug-sensitive epilepsy. In this study, although 12 of 17 patients after RF-TC had different degrees of recurrence during 8-28 months of followup, the number of AEDs used decreased from $2.4 \pm 0.7$ to 1.6 \pm 0.7. Therefore, SEEG-guided RF-TC has obvious characteristics of palliative treatment.

\section{Conclusions}

SEEG-guided RF-TC is a safe ablative procedure for drugresistant focal epilepsy; a reduction of seizures was observed in all patients who underwent RF-TC at our institution. The effect of RF-TC depends on the accuracy of the hypothesis of the SOZ. Limited by the scope of destruction, seizures often recur during follow-up. However, RF-TC can achieve the effect of palliative treatment in patients with epilepsy who refuse resection surgery or in those who cannot undergo resection surgery, that is, reduce the frequency of seizures and help improve the quality of life of patients. RF-TC does not affect surgical resection after epilepsy recurrence, and patients with a relapse can achieve freedom from seizures by undergoing resection surgery.

\section{Acknowledgments}

Funding: This study was supported by grants from the Natural Science Foundation of Tianjin City (No. 20JCYBJC00930, No. 20JCYBJC00920).

\section{Footnote}

Reporting Checklist: The authors have completed the STROBE reporting checklist. Available at https://atm. amegroups.com/article/view/10.21037/atm-21-6851/rc

Data Sharing Statement: Available at https://atm.amegroups. com/article/view/10.21037/atm-21-6851/dss

Conflicts of Interest: All authors have completed the ICMJE uniform disclosure form (available at https://atm. amegroups.com/article/view/10.21037/atm-21-6851/coif). The authors have no conflicts of interest to declare.

Ethical Statement: The authors are accountable for all aspects of the work in ensuring that questions related to the accuracy or integrity of any part of the work are appropriately investigated and resolved. All procedures performed in this study involving human participants were in accordance with the Declaration of Helsinki (as revised in 2013). The study was approved by ethics board of Tianjin Huanhu Hospital (No. 2021-059). Individual consent for this retrospective analysis was waived.

Open Access Statement: This is an Open Access article distributed in accordance with the Creative Commons Attribution-NonCommercial-NoDerivs 4.0 International License (CC BY-NC-ND 4.0), which permits the noncommercial replication and distribution of the article with the strict proviso that no changes or edits are made and the original work is properly cited (including links to both the formal publication through the relevant DOI and the license). See: https://creativecommons.org/licenses/by-nc-nd/4.0/.

\section{References}

1. Abou-Al-Shaar H, Brock AA, Kundu B, et al. Increased nationwide use of stereoencephalography for intracranial epilepsy electroencephalography recordings. J Clin Neurosci 2018;53:132-4.

2. Isnard J, Taussig D, Bartolomei F, et al. French guidelines on stereoelectroencephalography (SEEG). Neurophysiol Clin 2018;48:5-13.

3. Guénot M, Isnard J, Ryvlin P, et al. SEEG-guided RF thermocoagulation of epileptic foci: feasibility, safety, and preliminary results. Epilepsia 2004;45:1368-74.

4. Bartolomei F, Lagarde S, Scavarda D, et al. The role of the dorsal anterior insula in ecstatic sensation revealed by direct electrical brain stimulation. Brain Stimul 2019;12:1121-6.

5. Guekht A, Brodie M, Secco M, et al. The road to a World Health Organization global action plan on epilepsy and other neurological disorders. Epilepsia 2021;62:1057-63.

6. Rugg-Gunn F, Miserocchi A, McEvoy A. Epilepsy surgery. Pract Neurol 2020;20:4-14.

7. Bourdillon P, Rheims S, Catenoix H, et al. Surgical techniques: Stereoelectroencephalography-guided radiofrequency-thermocoagulation (SEEG-guided RFTC). Seizure 2020;77:64-8. 
8. Bourdillon P, Isnard J, Catenoix H, et al. Stereoelectro-encephalography-Guided Radiofrequency Thermocoagulation: From In Vitro and In Vivo Data to Technical Guidelines. World Neurosurg 2016;94:73-9.

9. Wang M, Zhang Y, Xue P, et al. Optimized SEEGguided radiofrequency thermocoagulation in the treatment of pediatric hypothalamic hamartomas. Seizure 2021;86:102-8.

10. Fan X, Shan Y, Lu C, et al. Optimized SEEG-guided radiofrequency thermocoagulation for mesial temporal lobe epilepsy with hippocampal sclerosis. Seizure 2019;71:304-11.

11. Cossu M, Cardinale F, Casaceli G, et al. Stereo-EEGguided radiofrequency thermocoagulations. Epilepsia 2017;58 Suppl 1:66-72.

12. Dimova P, de Palma L, Job-Chapron AS, et al. Radiofrequency thermocoagulation of the seizure-onset zone during stereoelectroencephalography. Epilepsia

Cite this article as: Wang L, Jin W, Zhang Y, Wang S, Li Q, Qin J, Li Z, Cheng Y, Feng K, Yin S. Stereoelectroencephalographyguided radiofrequency thermocoagulation in drug-resistant focal epilepsy. Ann Transl Med 2022;10(4):192. doi: 10.21037/atm21-6851
2017;58:381-92.

13. Kameyama S, Shirozu H, Masuda H, et al. MRI-guided stereotactic radiofrequency thermocoagulation for 100 hypothalamic hamartomas. J Neurosurg 2016;124:1503-12.

14. Wellmer J. Lesion focused radiofrequency thermocoagulation of bottom-of-sulcus focal cortical dysplasia type IIb: Conceptional considerations with regard to the epileptogenic zone. Epilepsy Res 2018;142:143-8.

15. Bourdillon P, Cucherat M, Isnard J, et al. Stereoelectroencephalography-guided radiofrequency thermocoagulation in patients with focal epilepsy: A systematic review and meta-analysis. Epilepsia 2018;59:2296-304.

16. Mirza FA, Hall JA. Radiofrequency Thermocoagulation in Refractory Focal Epilepsy: The Montreal Neurological Institute Experience. Can J Neurol Sci 2021;48:626-39.

(English Language Editor: L. Huleatt) 